\title{
22. PRELIMINARY REPORT ON PALEOMAGNETISM OF DEEP SEA DRILLING PROJECT LEG 4 SPECIMENS ${ }^{1}$
}

\author{
K. W. Henry and N. D. Opdyke, Lamont-Doherty Geological Observatory of \\ Columbia University, Palisades, New York
}

Paleomagnetic results from Leg 4 of the Deep Sea Drilling Project were disappointing. Poor sampling coverage, instrument failures, and generally inconsistent data led to inconclusive results.

Samples were treated in the same manner as for Legs 1, 2 and 3. Inclination, declination and intensity measurements were made of the sample natural remanent magnetization (NRM), after a magnetic cleaning in a peak alternating field of 50 oersteds. Due to a failure in the A. F.-demagnetizing apparatus, NRM measurements alone are available on samples from Sites 27, 30 and 31.

Unlike Leg 3, the sampling coverage on Leg 4 was too sparse to allow even tentative magnetic stratigraphics to be interpreted for the sections of continuous core. During Leg 3, oriented samples had been taken every 50 centimeters and at even closer intervals surrounding important paleontologic boundaries. Leg 4 samples, on the other hand, were at best 1.5 meters ( 5 feet) apart. Thus, when summary charts were constructed (Figures 7 through 10), the polarities of individual points rather than those of actual sections of core have been presented.

Paleomagnetic results from Legs 1, 2 and 3 showed that polarity interpretations had to be made relying

\footnotetext{
${ }^{1}$ Lamont-Doherty Geological Observatory Contribution No. 1431.
}

almost entirely upon magnetic inclination data. Declination values were not consistent, presumably due to the rotation of the core barrel during coring procedures.

For the previous legs this was not an insurmountable problem, as inclination values were quite high due to the latitude of the sites; and, although the internal numerical consistency of the values was very poor, inclination sign changes were taken to be fairly reliable indicators of polarity reversals. However, Leg 4 drilling sites were from very low latitudes and, thus, magnetic inclination values would be expected to be very low. This means that the data would have to have good internal consistency (better than any of the previous legs) to be reliably interpreted on inclination alone. Unfortunately, this is not the case. For example, Sites 23,24 and 25 were from latitudes of less than 7 degrees where expected inclination values based on an axial dipole are less than 14 degrees. By averaging the cleaned inclination values of the eleven samples that comprise the data from these three sites, the standard deviation around the mean is greater than 25 degrees. Therefore, a change of sign of the inclination is within this deviation without being due to a polarity reversal. Also, many inclination values are much higher than those expected from these latitudes and the reason for this is not known. Thus, reliability of the data is understandably questionable.

No conclusions were therefore drawn from the Leg 4 results except that reversals do seem to occur in all sampled geologic epochs. Actual results are presented in Tables 1 and 2, and Figures 1 through 10. 
TABLE 1

Summary of Magnetic Data, Leg 4

Sites 23 through 31 declination and inclination values and intensity in e.m.u./gm. are given for each sample for NRM and after cleaning in a field of 50 oersteds.

\begin{tabular}{|c|c|c|c|c|c|c|c|c|c|}
\hline \multirow[b]{2}{*}{ Hole } & \multirow[b]{2}{*}{ Core } & \multirow[b]{2}{*}{ Section } & \multirow{2}{*}{$\begin{array}{l}\text { Sampled at } \\
\text { (cm) }\end{array}$} & \multicolumn{3}{|c|}{ NRM } & \multicolumn{3}{|c|}{50 Oersted } \\
\hline & & & & Decl. & Incl. & Intensity emu/gm & Decl. & Incl. & Intensity emu/gm \\
\hline 23 & 3 & 2 & 16 & 258.0 & 41.7 & $1.123 \times 10^{-5}$ & 281.7 & 42.6 & $8.809 \times 10^{-6}$ \\
\hline 23 & 3 & 3 & 23 & 337.2 & -77.5 & $1.685 \times 10^{-5}$ & 321.6 & -59.6 & $1.508 \times 10^{-5}$ \\
\hline 23 & 4 & 1 & 10 & 80.7 & -78.8 & $8.878 \times 10^{-6}$ & 45.0 & -77.2 & $8.423 \times 10^{-6}$ \\
\hline 23 & 4 & 3 & 40 & 175.6 & -1.2 & $8.881 \times 10^{-6}$ & 199.0 & 14.1 & $1.390 \times 10^{-6}$ \\
\hline 23 & 5 & 1 & 22 & 138.2 & -29.9 & $7.420 \times 10^{-7}$ & 140.1 & -34.0 & $7.233 \times 10^{-7}$ \\
\hline 23 & 5 & 1 & 46 & 66.0 & -13.0 & $3.187 \times 10^{-7}$ & 53.7 & -31.7 & $1.074 \times 10^{-7}$ \\
\hline 24 & 4 & 2 & 13 & 199.5 & 10.0 & $2.524 \times 10^{-5}$ & 231.1 & 58.6 & $5.425 \times 10^{-6}$ \\
\hline 24 & 4 & 3 & 29 & 320.1 & -71.2 & $1.275 \times 10^{-7}$ & 314.7 & -79.0 & $9.297 \times 10^{-8}$ \\
\hline 24 & 4 & 4 & 6 & 215.4 & 17.6 & $2.129 \times 10^{-7}$ & 186.3 & -2.6 & $1.481 \times 10^{-7}$ \\
\hline $24 \mathrm{~A}$ & 3 & 1 & 86 & 132.0 & 53.7 & $1.163 \times 10^{-5}$ & 118.2 & 54.0 & $1.092 \times 10^{-5}$ \\
\hline $25 \mathrm{~A}$ & 1 & 1 & 87 & 194.9 & -3.6 & $8.549 \times 10^{-8}$ & 176.2 & 13.9 & $6.233 \times 10^{-8}$ \\
\hline 26 & 1 & 2 & 90 & 147.9 & 20.0 & $1.059 \times 10^{-5}$ & 140.2 & 22.0 & $8.400 \times 10^{-6}$ \\
\hline 26 & 1 & 3 & 138 & 210.6 & -3.8 & $4.637 \times 10^{-5}$ & 211.5 & -3.8 & $3.839 \times 10^{-5}$ \\
\hline 26 & 2 & 1 & 124 & 53.8 & -21.8 & $5.665 \times 10^{-6}$ & 39.5 & -15.8 & $6.601 \times 10^{-6}$ \\
\hline 26 & 2 & 2 & 10 & 110.8 & 10.0 & $1.852 \times 10^{-5}$ & 102.4 & 9.9 & $1.675 \times 10^{-5}$ \\
\hline 26 & 2 & 2 & 95 & 222.4 & 23.9 & $7.504 \times 10^{-6}$ & 249.5 & 23.9 & $5.540 \times 10^{-6}$ \\
\hline 26 & 3 & 2 & 20 & 195.6 & -25.6 & $2.934 \times 10^{-6}$ & 241.8 & -47.6 & $1.750 \times 10^{-6}$ \\
\hline 26 & 3 & 2 & 100 & 147.8 & 1.9 & $7.227 \times 10^{-6}$ & 132.4 & 8.9 & $5.130 \times 10^{-6}$ \\
\hline 26 & 5 & 1 & 13 & 177.8 & 11.5 & $1.485 \times 10^{-5}$ & 182.0 & -11.3 & $9.460 \times 10^{-6}$ \\
\hline 26 & 5 & 2 & 26 & 201.0 & 25.8 & $9.378 \times 10^{-6}$ & 209.9 & 25.7 & $5.470 \times 10^{-6}$ \\
\hline 26 & 5 & 2 & 90 & 164.7 & -0.7 & $2.175 \times 10^{-5}$ & 164.2 & 14.5 & $1.381 \times 10^{-5}$ \\
\hline 26 & 5 & 3 & 27 & 43.6 & 54.3 & $7.318 \times 10^{-6}$ & 30.1 & 38.5 & $7.387 \times 10^{-6}$ \\
\hline
\end{tabular}


TABLE 1 - Continued

\begin{tabular}{|c|c|c|c|c|c|c|c|c|c|}
\hline \multirow[b]{2}{*}{ Hole } & \multirow[b]{2}{*}{ Core } & \multirow[b]{2}{*}{ Section } & \multirow{2}{*}{$\begin{array}{l}\text { Sampled at } \\
\text { (cm) }\end{array}$} & \multicolumn{3}{|c|}{ NRM } & \multicolumn{3}{|c|}{50 Oersted } \\
\hline & & & & Decl. & Incl. & Intensity emu/gm & Decl. & Incl. & Intensity emu/gm \\
\hline 26 & 5 & 3 & 96 & 346.0 & 45.6 & $1.023 \times 10^{-5}$ & 339.1 & 37.7 & $9.714 \times 10^{-6}$ \\
\hline 26 & 5 & 4. & 87 & 96.8 & -6.0 & $1.546 \times 10^{-5}$ & 86.5 & -6.7 & $1.440 \times 10^{-4}$ \\
\hline 27 & 1 & 2 & 15 & 17.4 & 48.0 & $1.558 \times 10^{-6}$ & - & - & - \\
\hline 27 & 1 & 6 & 73 & 172.8 & 15.7 & $8.271 \times 10^{-7}$ & 151.6 & -30.0 & $7.629 \times 10^{-6}$ \\
\hline 27 & 2 & 1 & 86 & 177.4 & 48.6 & $1.349 \times 10^{-5}$ & & & \\
\hline 27 & 2 & 2 & 13 & 160.8 & 56.9 & $8.169 \times 10^{-6}$ & 204.4 & -3.4 & $3.655 \times 10^{-6}$ \\
\hline 27 & 2 & 3 & 21 & 154.7 & 42.7 & $6.472 \times 10^{-6}$ & 196.0 & 23.3 & $8.522 \times 10^{-6}$ \\
\hline 27 & 2 & 3 & 80 & 172.8 & 36.4 & $7.449 \times 10^{-6}$ & 110.0 & 70.3 & $5.967 \times 10^{-6}$ \\
\hline 27 & 3 & 1 & 47 & 109.7 & 23.9 & $9.405 \times 10^{-7}$ & 143.0 & 22.9 & $5.215 \times 10^{-6}$ \\
\hline 27 & 3 & 2 & 16 & 122.4 & 33.3 & $1.477 \times 10^{-6}$ & 205.3 & 58.0 & $1.072 \times 10^{-5}$ \\
\hline 27 & 4 & 1 & 35 & 197.4 & 29.3 & $7.186 \times 10^{-6}$ & 254.2 & 2.7 & $2.261 \times 10^{-6}$ \\
\hline 27 & 4 & 2 & 4 & 70.6 & 4.2 & $3.837 \times 10^{-7}$ & 85.4 & -12.7 & $2.222 \times 10^{-6}$ \\
\hline 27 & 4 & 3 & 16 & 292.8 & 52.0 & $7.262 \times 10^{-7}$ & 209.8 & 23.0 & $5.593 \times 10^{-6}$ \\
\hline 27 & 5 & 1 & 19 & 144.3 & 6.7 & $4.318 \times 10^{-7}$ & 203.3 & 37.6 & $4.221 \times 10^{-6}$ \\
\hline 27 & 5 & 2 & 39 & 79.3 & 42.7 & $2.809 \times 10^{-7}$ & & & \\
\hline 27 & 5 & 3 & 25 & 215.0 & 17.2 & $1.094 \times 10^{-7}$ & 258.3 & -34.7 & $2.358 \times 10^{-5}$ \\
\hline $27 \mathrm{~A}$ & 1 & 1 & 29 & 96.5 & 9.0 & $6.815 \times 10^{-6}$ & 193.8 & 27.9 & $5.904 \times 10^{-6}$ \\
\hline $27 \mathrm{~A}$ & 1 & 1 & 77 & 188.3 & 62.7 & $5.189 \times 10^{-6}$ & 242.5 & 60.3 & $3.492 \times 10^{-6}$ \\
\hline $27 \mathrm{~A}$ & 1 & 4 & 15 & 157.8 & 24.1 & $1.054 \times 10^{-5}$ & & & \\
\hline $27 \mathrm{~A}$ & 1 & 6 & 18 & 175.3 & 21.4 & $6.511 \times 10^{-6}$ & & & \\
\hline $27 \mathrm{~A}$ & 1 & 6 & 90 & 158.3 & 43.0 & $3.948 \times 10^{-6}$ & & & \\
\hline $27 \mathrm{~A}$ & 2 & 3 & 25 & 151.6 & -30.0 & $7.629 \times 10^{-6}$ & & & \\
\hline $27 \mathrm{~A}$ & 2 & 3 & 73 & 114.8 & 48.9 & $3.296 \times 10^{-6}$ & & & \\
\hline $27 \mathrm{~A}$ & 3 & 1 & 132 & 204.4 & -3.4 & $3.655 \times 10^{-6}$ & & & \\
\hline $27 \mathrm{~A}$ & 3 & 2 & 22 & 196.0 & 23.3 & $8.528 \times 10^{-6}$ & & & \\
\hline $27 \mathrm{~A}$ & 3 & 3 & 30 & 110.0 & 70.3 & $5.967 \times 10^{-6}$ & & & \\
\hline $27 \mathrm{~A}$ & 4 & 1 & 34 & 143.0 & 22.9 & $5.215 \times 10^{-6}$ & & & \\
\hline
\end{tabular}


TABLE 1 - Continued

\begin{tabular}{|c|c|c|c|c|c|c|c|c|c|}
\hline \multirow[b]{2}{*}{ Hole } & \multirow[b]{2}{*}{ Core } & \multirow[b]{2}{*}{ Section } & \multirow{2}{*}{$\begin{array}{l}\text { Sampled at } \\
(\mathrm{cm})\end{array}$} & \multicolumn{3}{|c|}{ NRM } & \multicolumn{3}{|c|}{50 Oersted } \\
\hline & & & & Decl. & Incl. & Intensity emu/gm & Decl. & Incl. & Intensity emu/gm \\
\hline $27 \mathrm{~A}$ & 4 & 2 & 49 & 205.3 & 58.0 & $1.072 \times 10^{-5}$ & & & \\
\hline $27 \mathrm{~A}$ & 4 & 3 & 15 & 129.8 & 72.5 & $3.592 \times 10^{-6}$ & & & \\
\hline $27 \mathrm{~A}$ & 4 & 3 & 22 & 254.2 & 2.7 & $2.261 \times 10^{-6}$ & & & \\
\hline $27 \mathrm{~A}$ & 4 & 4 & 33 & 85.4 & -12.7 & $2.222 \times 10^{-6}$ & & & \\
\hline $27 \mathrm{~A}$ & 4 & 5 & 35 & 209.8 & 23.0 & $5.593 \times 10^{-6}$ & & & \\
\hline $27 \mathrm{~A}$ & 4 & 6 & 34 & 147.7 & 19.4 & $5.402 \times 10^{-6}$ & & & \\
\hline $27 \mathrm{~A}$ & 5 & 1 & 32 & 203.2 & 37.6 & $4.281 \times 10^{-6}$ & & & \\
\hline $27 \mathrm{~A}$ & 5 & 2 & 46 & 258.3 & -34.7 & $2.358 \times 10^{-5}$ & & & \\
\hline $27 \mathrm{~A}$ & 5 & 3 & 22 & 163.6 & 55.4 & - & & & \\
\hline $27 \mathrm{~A}$ & 5 & 4 & 57 & 193.8 & 27.9 & $5.904 \times 10^{-6}$ & & & \\
\hline $27 \mathrm{~A}$ & 5 & 5 & 84 & 242.5 & 60.3 & $3.498 \times 10^{-6}$ & & & \\
\hline 28 & 2 & 2 & 10 & 176.9 & -0.9 & $1.571 \times 10^{-5}$ & 171.9 & -19.5 & $1.020 \times 10^{-5}$ \\
\hline 28 & 2 & 3 & 16 & 222.8 & 37.2 & $8.576 \times 10^{-6}$ & 240.4 & 24.1 & $5.849 \times 10^{-6}$ \\
\hline 28 & 2 & 4 & 18 & 237.6 & 38.4 & $1.331 \times 10^{-5}$ & 259.0 & 16.3 & $5.636 \times 10^{-6}$ \\
\hline 28 & 3 & 1 & 11 & 235.9 & 22.0 & $1.173 \times 10^{-7}$ & 84.7 & 76.6 & $1.631 \times 10^{-7}$ \\
\hline 28 & 3 & 2 & 36 & 36.9 & 40.0 & $3.386 \times 10^{-8}$ & 29.9 & -40.5 & $8.412 \times 10^{-8}$ \\
\hline 28 & 3 & 3 & 42 & 162.3 & 40.5 & $1.140 \times 10^{-7}$ & 77.0 & 47.7 & $7.705 \times 10^{-8}$ \\
\hline 28 & 3 & 4 & 57 & 110.6 & -47.4 & $1.628 \times 10^{-7}$ & 107.4 & -61.5 & $1.171 \times 10^{-7}$ \\
\hline 28 & 3 & 5 & 11 & 214.3 & -36.7 & $1.188 \times 10^{-7}$ & 247.3 & -51.6 & $7.919 \times 10^{-8}$ \\
\hline 28 & 3 & 6 & 24 & 35.5 & -42.1 & $2.909 \times 10^{-8}$ & 347.7 & -58.1 & $1.017 \times 10^{-7}$ \\
\hline 29 & 1 & 2 & 20 & 155.3 & 34.7 & $2.961 \times 10^{-6}$ & 145.8 & 48.4 & $1.751 \times 10^{-6}$ \\
\hline 29 & 1 & 3 & 30 & 132.9 & -65.2 & & 101.9 & 69.9 & \\
\hline 29 & 1 & 3 & 62 & 158.3 & 57.3 & $5.518 \times 10^{-6}$ & 152.5 & 64.6 & $4.077 \times 10^{-6}$ \\
\hline 29 & 2 & 1 & 52 & 177.1 & 54.8 & $7.579 \times 10^{-6}$ & 166.9 & 64.6 & $5.410 \times 10^{-6}$ \\
\hline 29 & 2 & 2 & 40 & 178.4 & 29.9 & $2.713 \times 10^{-6}$ & 176.0 & 40.3 & $1.748 \times 10^{-6}$ \\
\hline 29 & 2 & 3 & 50 & 142.5 & 70.3 & $3.703 \times 10^{-6}$ & 102.4 & 74.9 & $3.054 \times 10^{-6}$ \\
\hline
\end{tabular}


TABLE 1 - Continued

\begin{tabular}{|c|c|c|c|c|c|c|c|c|c|}
\hline \multirow[b]{2}{*}{ Hole } & \multirow[b]{2}{*}{ Core } & \multirow[b]{2}{*}{ Section } & \multirow{2}{*}{$\begin{array}{l}\text { Sampled at } \\
(\mathrm{cm})\end{array}$} & \multicolumn{3}{|c|}{ NRM } & \multicolumn{3}{|c|}{50 Oersted } \\
\hline & & & & Decl. & Incl. & Intensity emu/gm & Decl. & Incl. & Intensity emu/gm \\
\hline 29 & 4 & 1 & 109 & 130.0 & 64.9 & $1.286 \times 10^{-5}$ & 109.7 & 67.5 & $1.013 \times 10^{-5}$ \\
\hline 29 & 4 & 2 & 53 & 299.8 & 43.3 & $9.980 \times 10^{-5}$ & 303.4 & 41.1 & $9.575 \times 10^{-5}$ \\
\hline 29 & 4 & 3 & 48 & 85.8 & 77.3 & $1.192 \times 10^{-5}$ & 65.5 & 75.0 & $9.315 \times 10^{-6}$ \\
\hline 29 & 4 & 4 & 16 & 54.1 & 60.7 & $5.987 \times 10^{-6}$ & 36.4 & 59.1 & $4.681 \times 10^{-6}$ \\
\hline 29 & 5 & 1 & 122 & 323.1 & 21.3 & $6.885 \times 10^{-6}$ & 328.2 & 14.8 & $7.034 \times 10^{-6}$ \\
\hline 29 & 6 & 1 & 105 & 139.4 & 5.1 & $3.353 \times 10^{-5}$ & 133.5 & 4.3 & $2.447 \times 10^{-5}$ \\
\hline 29 & 7 & 1 & 137 & 120.1 & 38.8 & $4.978 \times 10^{-6}$ & 79.8 & 13.4 & $4.470 \times 10^{-6}$ \\
\hline 29 & 8 & $1 \mathrm{~B}$ & 118 & 139.5 & 2.0 & $1.341 \times 10^{-6}$ & 120.3 & 35.8 & $4.783 \times 10^{-7}$ \\
\hline 29 & 9 & 1 & 47 & 253.6 & -18.4 & - & 293.4 & -47.6 & - \\
\hline 29 & 9 & 2 & 48 & 196.9 & 33.1 & $1.602 \times 10^{-6}$ & 220.9 & 18.0 & $7.622 \times 10^{-7}$ \\
\hline 29 & 9 & 3 & 18 & 13.1 & -9.3 & $3.368 \times 10^{-7}$ & 23.5 & -27.7 & $1.408 \times 10^{-6}$ \\
\hline 29 & 9 & 4 & 35 & 79.4 & 13.4 & $3.224 \times 10^{-6}$ & 65.9 & 7.9 & $2.342 \times 10^{-6}$ \\
\hline 29 & 9 & 5 & 21 & 216.6 & 6.3 & - & 251.7 & -7.8 & - \\
\hline 29 & 9 & 6 & 33 & 225.8 & -32.2 & $1.528 \times 10^{-6}$ & 298.6 & -76.1 & $2.147 \times 10^{-6}$ \\
\hline 29 & 10 & 1 & 61 & 351.3 & -8.7 & $9.630 \times 10^{-6}$ & 344.0 & 6.2 & $7.961 \times 10^{-6}$ \\
\hline 29 & 10 & 2 & 39 & 231.8 & 20.3 & $3.435 \times 10^{-6}$ & 255.7 & 13.5 & $2.580 \times 10^{-6}$ \\
\hline 29 & 10 & 3 & 29 & 240.7 & 3.7 & $2.220 \times 10^{-6}$ & 273.9 & -8.9 & $2.324 \times 10^{-6}$ \\
\hline 29 & 10 & 4 & 34 & 189.4 & 38.7 & $1.305 \times 10^{-6}$ & 308.7 & 40.0 & $1.301 \times 10^{-7}$ \\
\hline 29 & 10 & 5 & 33 & 325.0 & 39.7 & $1.569 \times 10^{-6}$ & 336.8 & 11.3 & $1.279 \times 10^{-5}$ \\
\hline 29 & 11 & 1 & 140 & 73.0 & -30.7 & $2.006 \times 10^{-5}$ & 75.6 & -32.3 & $1.854 \times 10^{-5}$ \\
\hline 29 & 12 & 1 & 21 & 344.5 & 59.5 & $4.234 \times 10^{-6}$ & 340.4 & 31.4 & $3.563 \times 10^{-6}$ \\
\hline 29 & 12 & 2 & 35 & 239.3 & 20.3 & $1.552 \times 10^{-6}$ & 289.3 & 27.7 & $5.121 \times 10^{-7}$ \\
\hline 29 & 12 & 3 & 25 & 42.7 & 46.6 & $7.290 \times 10^{-6}$ & 35.8 & 39.2 & $5.254 \times 10^{-6}$ \\
\hline 29 & 12 & 4 & 32 & 156.4 & 28.3 & $4.252 \times 10^{-6}$ & 143.4 & 22.5 & $2.206 \times 10^{-6}$ \\
\hline 29 & 12 & 5 & 27 & 177.9 & -11.4 & $5.903 \times 10^{-6}$ & 182.1 & -21.6 & $2.709 \times 10^{-6}$ \\
\hline 29 & 12 & 6 & 36 & 97.0 & 36.5 & $4.418 \times 10^{-6}$ & 79.2 & 27.0 & - \\
\hline 29 & 14 & 1 & 31 & 181.6 & 32.8 & $1.217 \times 10^{-6}$ & 196.7 & 32.0 & $2.093 \times 10^{-7}$ \\
\hline
\end{tabular}




\begin{tabular}{|c|c|c|c|c|c|c|c|c|c|}
\hline \multirow[b]{2}{*}{ Hole } & \multirow[b]{2}{*}{ Core } & \multirow[b]{2}{*}{ Section } & \multirow{2}{*}{$\begin{array}{l}\text { Sampled at } \\
\text { (cm) }\end{array}$} & \multicolumn{3}{|c|}{ NRM } & \multicolumn{3}{|c|}{50 Oersted } \\
\hline & & & & Decl. & Incl. & Intensity emu/gm & Decl. & Incl. & Intensity $\mathrm{emu} / \mathrm{gm}$ \\
\hline 29 & 14 & 2 & 36 & 197.5 & 4.1 & $1.426 \times 10^{-6}$ & 341.8 & 21.6 & $8.332 \times 10^{-7}$ \\
\hline 29 & 14 & 3 & 32 & 292.5 & -23.0 & $2.504 \times 10^{-6}$ & 308.5 & -28.5 & $3.019 \times 10^{-6}$ \\
\hline 29 & 14 & 4 & 35 & 88.1 & 1.8 & $2: 151 \times 10^{-6}$ & 76.0 & -5.9 & $2.641 \times 10^{-6}$ \\
\hline 29 & 14 & 5 & 28 & 119.0 & -14.0 & $2.759 \times 10^{-6}$ & 110.5 & -22.9 & $2.563 \times 10^{-6}$ \\
\hline 29 & 14 & 6 & 30 & 201.9 & -14.9 & $3.218 \times 10^{-6}$ & 207.3 & -26.1 & $2.701 \times 10^{-6}$ \\
\hline 29 & 15 & 1 & 32 & 354.6 & -47.7 & $1.192 \times 10^{-6}$ & 4.2 & -44.4 & $1.481 \times 10^{-6}$ \\
\hline 29 & 15 & 2 & 32 & 268.7 & 8.2 & $1.129 \times 10^{-6}$ & 301.7 & -8.3 & $1.228 \times 10^{-6}$ \\
\hline 29 & 15 & 3 & 34 & 230.8 & -27.4 & $4.758 \times 10^{-6}$ & 241.0 & -34.0 & $4.059 \times 10^{-6}$ \\
\hline 29 & 15 & 4 & 29 & 69.6 & -19.4 & - & 55.1 & -23.2 & - \\
\hline 29 & 15 & 5 & 36 & 318.2 & -53.4 & - & 341.3 & -41.9 & - \\
\hline 29 & 15 & 6 & 45 & 100.8 & -30.1 & $1.331 \times 10^{-6}$ & 79.2 & -36.8 & $1.447 \times 10^{-6}$ \\
\hline 29 & 16 & 1 & 46 & 187.9 & -17.3 & $4.835 \times 10^{-6}$ & 355.6 & -21.4 & $3.930 \times 10^{-6}$ \\
\hline 29 & 16 & 2 & 33 & 155.2 & -14.1 & $3.022 \times 10^{-6}$ & 151.4 & -17.5 & $2.524 \times 10^{-6}$ \\
\hline 29 & 16 & 3 & 46 & 232.8 & -18.4 & $5.679 \times 10^{-6}$ & 241.5 & -26.9 & $4.807 \times 10^{-6}$ \\
\hline 29 & 16 & 4 & 38 & 354.3 & -62.2 & $3.515 \times 10^{-6}$ & 345.7 & -57.9 & $3.901 \times 10^{-6}$ \\
\hline 29 & 16 & 5 & 32 & 101.6 & -33.3 & $1.198 \times 10^{-6}$ & 48.3 & -43.2 & $1.558 \times 10^{-6}$ \\
\hline 29 & 16 & 6 & 33 & 330.2 & -43.0 & $1.788 \times 10^{-6}$ & 341.3 & -29.6 & $2.345 \times 10^{-6}$ \\
\hline 29 & 17 & 1 & 31 & 240.6 & -76.8 & $2.731 \times 10^{-6}$ & 313.1 & -74.2 & $2.723 \times 10^{-6}$ \\
\hline 29 & 17 & 2 & 32 & 232.7 & 2.2 & $4.111 \times 10^{-6}$ & 249.9 & -8.0 & $2.902 \times 10^{-6}$ \\
\hline 29 & 17 & 3 & 31 & 163.2 & 7.3 & $1.573 \times 10^{-6}$ & 157.4 & -3.8 & $3.219 \times 10^{-6}$ \\
\hline 29 & 17 & 4 & 32 & 157.7 & -22.0 & $3.883 \times 10^{-6}$ & 153.2 & -31.7 & $2.527 \times 10^{-6}$ \\
\hline 29 & 17 & 5 & 32 & 196.4 & 9.3 & - & - & - & - \\
\hline 29 & 18 & 2 & 42 & 158.1 & 9.3 & $1.832 \times 10^{-6}$ & 133.5 & 66.7 & $3.622 \times 10^{-7}$ \\
\hline $29 \mathrm{~A}$ & 2 & 1 & 40 & 231.2 & 43.6 & $3.306 \times 10^{-5}$ & - & - & - \\
\hline $29 \mathrm{~A}$ & 5 & 1 & 56 & 178.9 & 37.7 & $2.123 \times 10^{-5}$ & - & - & - \\
\hline $29 \mathrm{~B}$ & 1 & 1 & 33 & 144.7 & 11.4 & $1.226 \times 10^{-5}$ & 138.2 & 8.8 & $1.001 \times 10^{-5}$ \\
\hline 29B & 1 & 4 & 131 & 233.2 & 21.3 & $1.119 \times 10^{-5}$ & 249.3 & 28.7 & $8.826 \times 10^{-6}$ \\
\hline
\end{tabular}


TABLE 1 - Continued

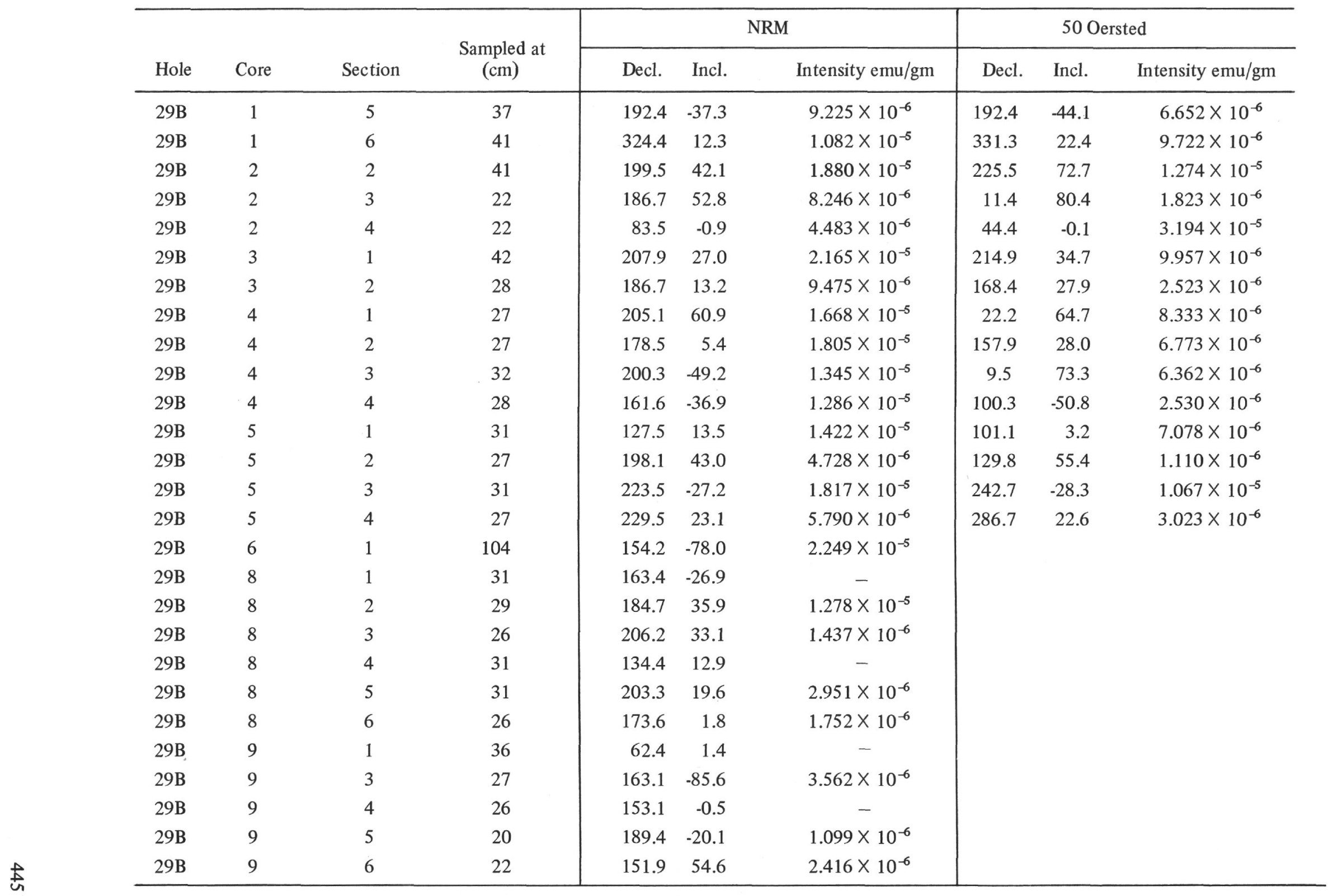


TABLE 1 - Continued

\begin{tabular}{|c|c|c|c|c|c|c|c|c|c|}
\hline \multirow[b]{2}{*}{ Hole } & \multirow[b]{2}{*}{ Core } & \multirow[b]{2}{*}{ Section } & \multirow{2}{*}{$\begin{array}{l}\text { Sampled at } \\
(\mathrm{cm})\end{array}$} & \multicolumn{3}{|c|}{ NRM } & \multicolumn{3}{|c|}{50 Oersted } \\
\hline & & & & Decl. & Incl. & Intensity emu/gm & Decl. & Incl. & Intensity emu/gm \\
\hline 29B & 0 & 2 & 27 & 179.0 & -13.9 & $3.344 \times 10^{-6}$ & \multirow{16}{*}{$\begin{array}{l}197.6 \\
197.7 \\
183.9 \\
191.3\end{array}$} & \multirow[b]{6}{*}{53.7} & \multirow{27}{*}{$\begin{array}{l}5.644 \times 10^{-7} \\
7.011 \times 10^{-7} \\
2.755 \times 10^{-7} \\
1.210 \times 10^{-5}\end{array}$} \\
\hline $29 \mathrm{~B}$ & 0 & 3 & 32 & 191.7 & 4.3 & $3.979 \times 10^{-6}$ & & & \\
\hline $29 B$ & 0 & 4 & 33 & 209.3 & 2.5 & $3.861 \times 10^{-6}$ & & & \\
\hline $29 \mathrm{~B}$ & 0 & 5 & 44 & 135.2 & -60.8 & $2.190 \times 10^{-6}$ & & & \\
\hline 30 & 2 & 2 & 20 & 154.7 & 64.3 & $1.928 \times 10^{-5}$ & & & \\
\hline 30 & 2 & 3 & 31 & 124.3 & 67.0 & $2.799 \times 10^{-5}$ & & & \\
\hline 30 & 2 & 4 & 32 & 240.2 & -71.0 & $5.206 \times 10^{-6}$ & & 26.9 & \\
\hline 30 & 3 & 2 & 31 & 222.1 & 52.2 & $8.076 \times 10^{-6}$ & & -11.6 & \\
\hline 30 & 4 & 1 & 110 & 157.5 & 62.4 & $2.009 \times 10^{-5}$ & & 47.6 & \\
\hline 30 & 4 & 2 & 22 & 124.9 & 69.7 & $3.293 \times 10^{-6}$ & & & \\
\hline 30 & 5 & 1 & 42 & 197.6 & 53.7 & $5.644 \times 10^{-7}$ & & & \\
\hline 30 & 5 & 2 & 29 & 197.7 & 26.9 & $7.011 \times 10^{-7}$ & & & \\
\hline 30 & 6 & 1 & 31 & 183.9 & -11.6 & $2.755 \times 10^{-7}$ & & & \\
\hline 30 & 6 & 2 & 36 & 199.1 & 26.5 & $4.092 \times 10^{-7}$ & & & \\
\hline 30 & 6 & 3 & 21 & 191.3 & 47.6 & $1.210 \times 10^{-5}$ & & & \\
\hline 30 & 7 & 1 & 68 & 166.8 & 75.2 & $7.577 \times 10^{-6}$ & & & \\
\hline 31 & 1 & 1 & 41 & 196.1 & 50.7 & $3.829 \times 10^{-6}$ & \multirow[t]{11}{*}{199.8} & \multirow[t]{11}{*}{75.1} & \\
\hline 31 & 1 & 5 & 28 & 305.5 & 71.6 & $4.068 \times 10^{-6}$ & & & \\
\hline 31 & 3 & 3 & 36 & 267.1 & 71.5 & $3.713 \times 10^{-5}$ & & & \\
\hline 31 & 3 & 5 & 26 & 186.7 & 57.0 & $1.460 \times 10^{-5}$ & & & \\
\hline 31 & 4 & 1 & 78 & 220.7 & 7.7 & $4.721 \times 10^{-5}$ & & & \\
\hline 31 & 6 & 1 & 32 & 92.8 & -32.3 & $1.862 \times 10^{-5}$ & & & \\
\hline 31 & 6 & 2 & 31 & 99.9 & 75.0 & $1.808 \times 10^{-5}$ & & & \\
\hline 31 & 7 & 1 & 65 & 143.5 & 75.2 & $1.557 \times 10^{-5}$ & & & \\
\hline 31 & 7 & 1 & 140 & 199.8 & 75.1 & $1.100 \times 10^{-5}$ & & & \\
\hline 31 & 8 & 1 & 58 & 165.0 & 20.4 & $2.448 \times 10^{-7}$ & & & \\
\hline 31 & 8 & 2 & 27 & 196.3 & 72.6 & $1.683 \times 10^{-6}$ & & & \\
\hline
\end{tabular}


TABLE 2

Summary of Magnetic Data

Alternating Field Demagnetization Results on Pilot Specimens

\begin{tabular}{|c|c|c|c|c|c|c|c|}
\hline Hole & Core & Section & $\begin{array}{l}\text { Sampled at } \\
\text { (cm) }\end{array}$ & $\begin{array}{l}\text { Peak Field } \\
\text { in Oersteds }\end{array}$ & Decl. & Incl. & Intensity $(\mathrm{emu} / \mathrm{gm})$ \\
\hline \multirow[t]{5}{*}{23} & 3 & 2 & 16 & NRM & 258.0 & 41.7 & $1.183 \times 10^{-5}$ \\
\hline & & & & 0500 & 281.7 & 42.6 & $8.809 \times 10^{-6}$ \\
\hline & & & & 1000 & 283.0 & -44.7 & $6.732 \times 10^{-6}$ \\
\hline & & & & 1500 & 275.8 & 42.4 & $4.659 \times 10^{-6}$ \\
\hline & & & & 2000 & 280.7 & 29.5 & $1.981 \times 10^{-6}$ \\
\hline \multirow[t]{5}{*}{24} & 4 & 2 & 13 & NRM & 199.5 & 10.0 & $2.524 \times 10^{-5}$ \\
\hline & & & & 0500 & 231.1 & 58.6 & $5.485 \times 10^{-6}$ \\
\hline & & & & 1000 & 91.5 & 55.4 & $3.739 \times 10^{-6}$ \\
\hline & & & & 1500 & 249.0 & -39.0 & $2.615 \times 10^{-6}$ \\
\hline & & & & 2000 & 217.6 & 57.0 & $5.630 \times 10^{-6}$ \\
\hline \multirow[t]{5}{*}{$24 \mathrm{~A}$} & 3 & 1 & 86 & NRM & 132.0 & 53.7 & $1.163 \times 10^{-5}$ \\
\hline & & & & 0500 & 118.2 & 54.0 & $1.092 \times 10^{-5}$ \\
\hline & & & & 1000 & 107.8 & 52.8 & $8.951 \times 10^{-6}$ \\
\hline & & & & 1500 & 114.4 & 48.6 & $7.822 \times 10^{-6}$ \\
\hline & & & & 2000 & 120.4 & 45.8 & $7.355 \times 10^{-6}$ \\
\hline \multirow[t]{5}{*}{$25 \mathrm{~A}$} & 1 & 1 & 87 & NRM & 194.9 & -3.6 & $8.549 \times 10^{-8}$ \\
\hline & & & & 0500 & 176.2 & 13.9 & $6.233 \times 10^{-8}$ \\
\hline & & & & 1000 & 169.6 & 9.1 & $4.971 \times 10^{-7}$ \\
\hline & & & & 1500 & 193.7 & 30.6 & $4.862 \times 10^{-7}$ \\
\hline & & & & 2000 & 161.4 & 9.3 & $2.780 \times 10^{-7}$ \\
\hline \multirow[t]{4}{*}{26} & 1 & 2 & 90 & NRM & 147.9 & 20.0 & $1.059 \times 10^{-5}$ \\
\hline & & & & 0500 & 140.2 & 22.0 & $8.400 \times 10^{-6}$ \\
\hline & & & & 1000 & 141.3 & 22.6 & $7.144 \times 10^{-6}$ \\
\hline & & & & 1500 & 140.7 & 23.5 & $6.018 \times 10^{-6}$ \\
\hline
\end{tabular}


TABLE 2 - Continued

\begin{tabular}{|c|c|c|c|c|c|c|c|}
\hline Hole & Core & Section & $\begin{array}{l}\text { Sampled at } \\
\text { (cm) }\end{array}$ & $\begin{array}{l}\text { Peak Field } \\
\text { in Oersteds }\end{array}$ & Decl. & Incl. & Intensity $(\mathrm{emu} / \mathrm{gm})$ \\
\hline 26 & 1 & 2 & 90 & 2000 & 146.4 & 20.2 & $4.841 \times 10^{-6}$ \\
\hline \multirow[t]{5}{*}{28} & 2 & 2 & 10 & NRM & 176.9 & -0.9 & $1.571 \times 10^{-5}$ \\
\hline & & & & 0500 & 171.9 & -19.5 & $1.020 \times 10^{-5}$ \\
\hline & & & & 1000 & 164.7 & -26.8 & $6.976 \times 10^{-6}$ \\
\hline & & & & 1500 & 170.7 & -25.3 & $4.128 \times 10^{-6}$ \\
\hline & & & & 2000 & 164.6 & 2.3 & $3.709 \times 10^{-6}$ \\
\hline \multirow[t]{5}{*}{29} & 1 & 3 & 30 & NRM & 132.9 & -65.2 & \\
\hline & & & & 0500 & 101.9 & 69.9 & \\
\hline & & & & 1000 & 112.0 & 64.1 & \\
\hline & & & & 1500 & 117.8 & 69.9 & \\
\hline & & & & 2000 & 115.0 & 55.7 & \\
\hline
\end{tabular}




\section{ALTERNATING FIELD DEMAGNETIZATION CURVES}

Figures 1 through 6

Alternating Field Demagnetization Curves for pilot specimens. Intensity is plotted as percent of NRM intensity. 


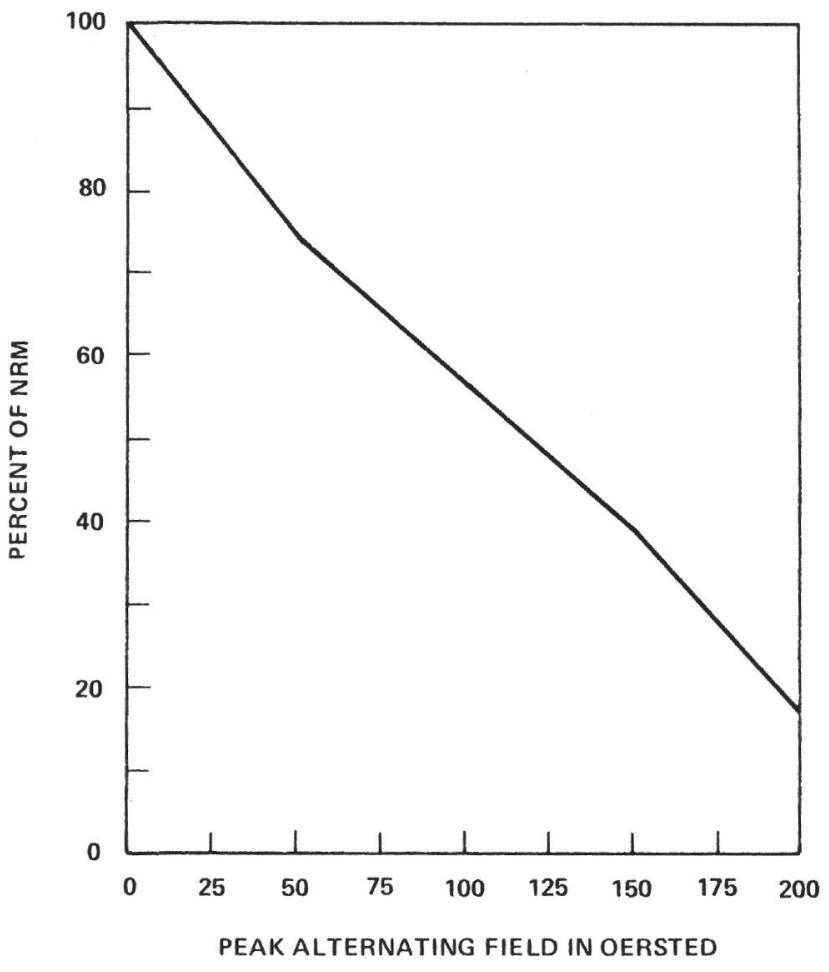

Figure 1. Sample 23-3-2, $16 \mathrm{~cm}$.

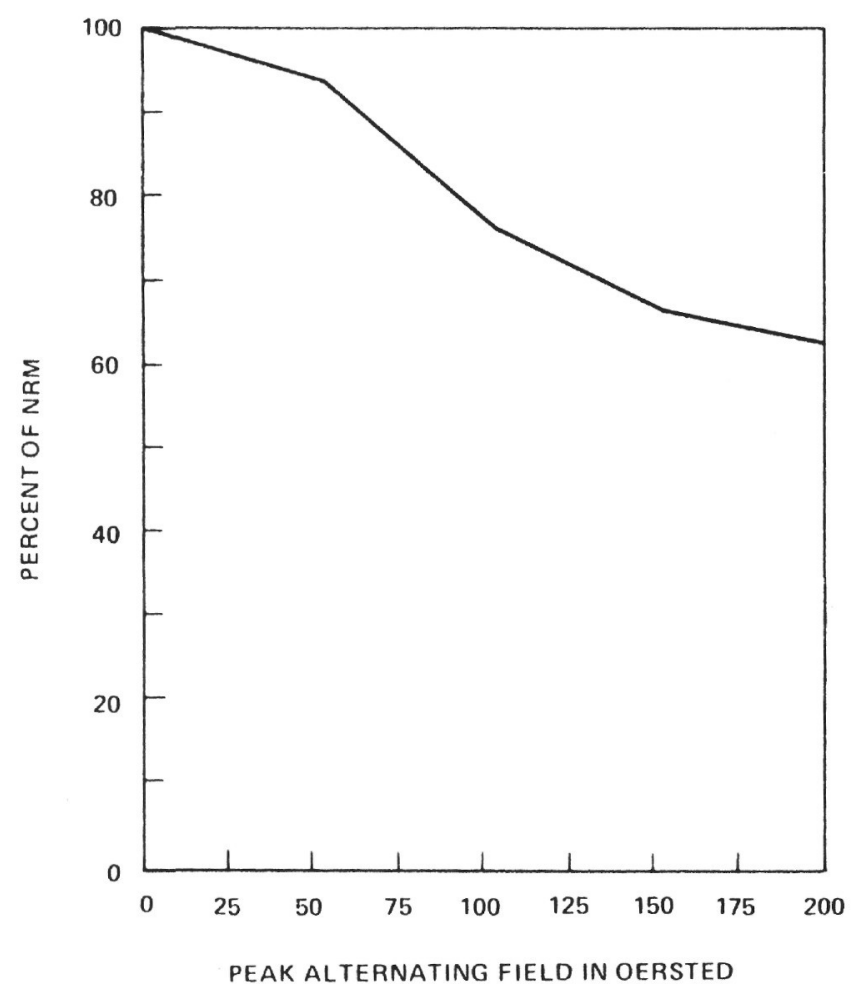

Figure 3. Sample 24A-3-1, $36 \mathrm{~cm}$.

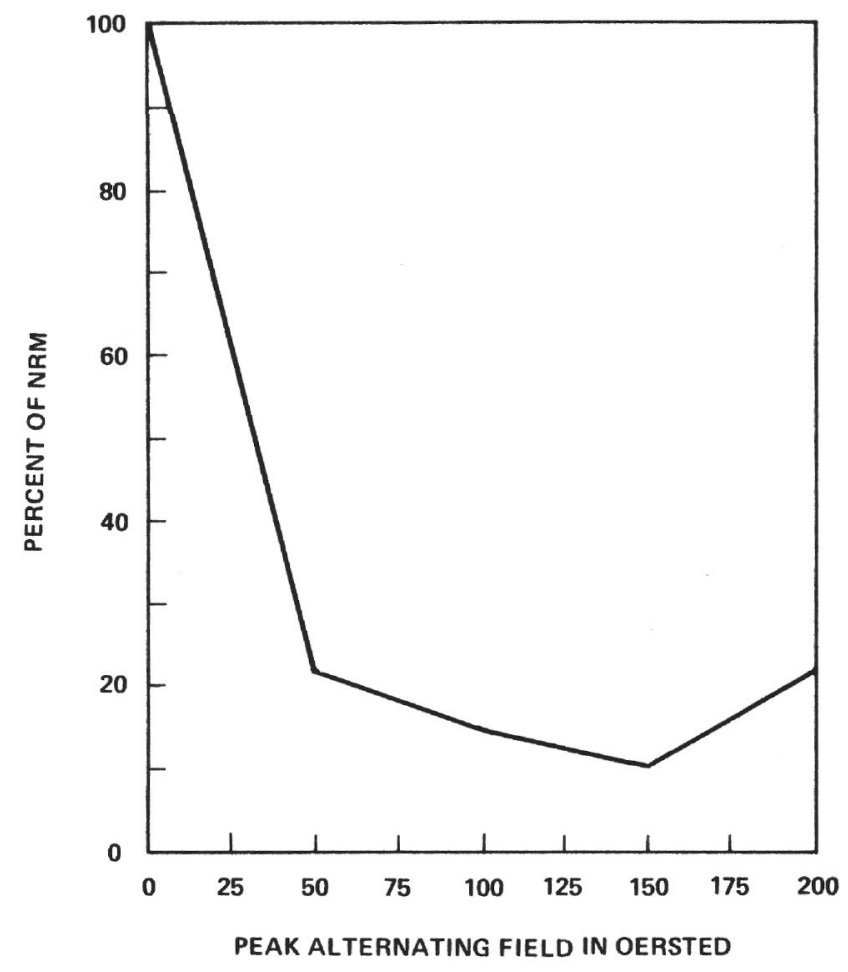

Figure 2. Sample 24-4-2, $13 \mathrm{~cm}$.

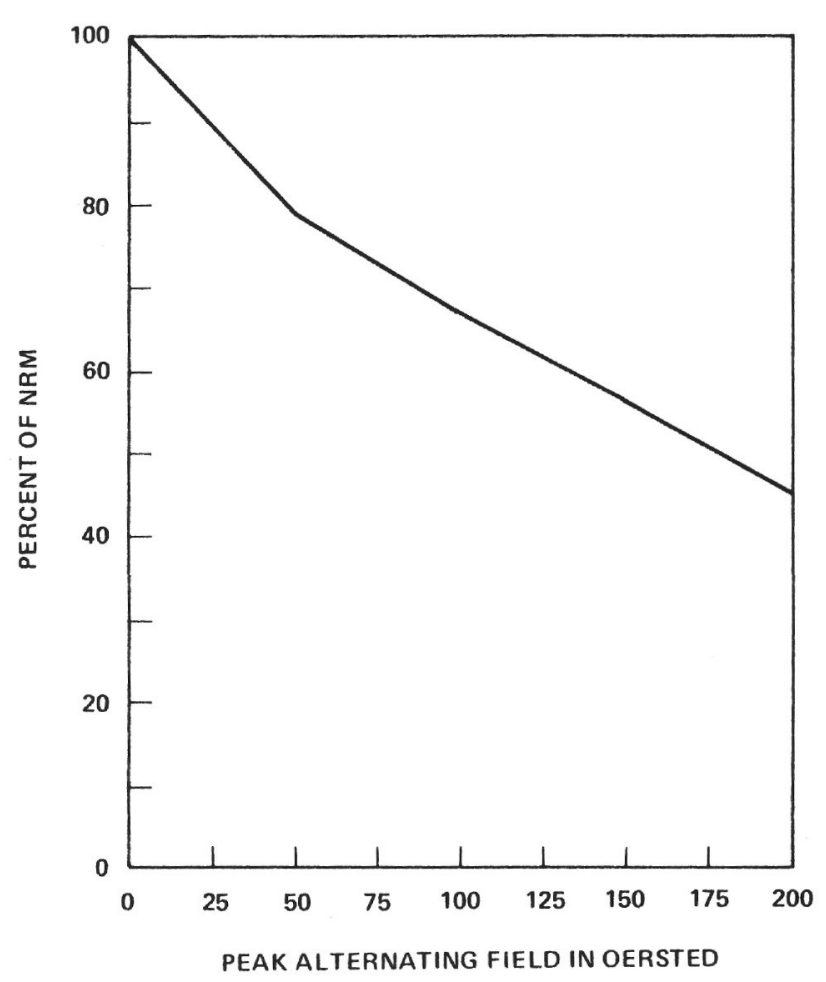

Figure 4. Sample 26-1-2, $90 \mathrm{~cm}$. 


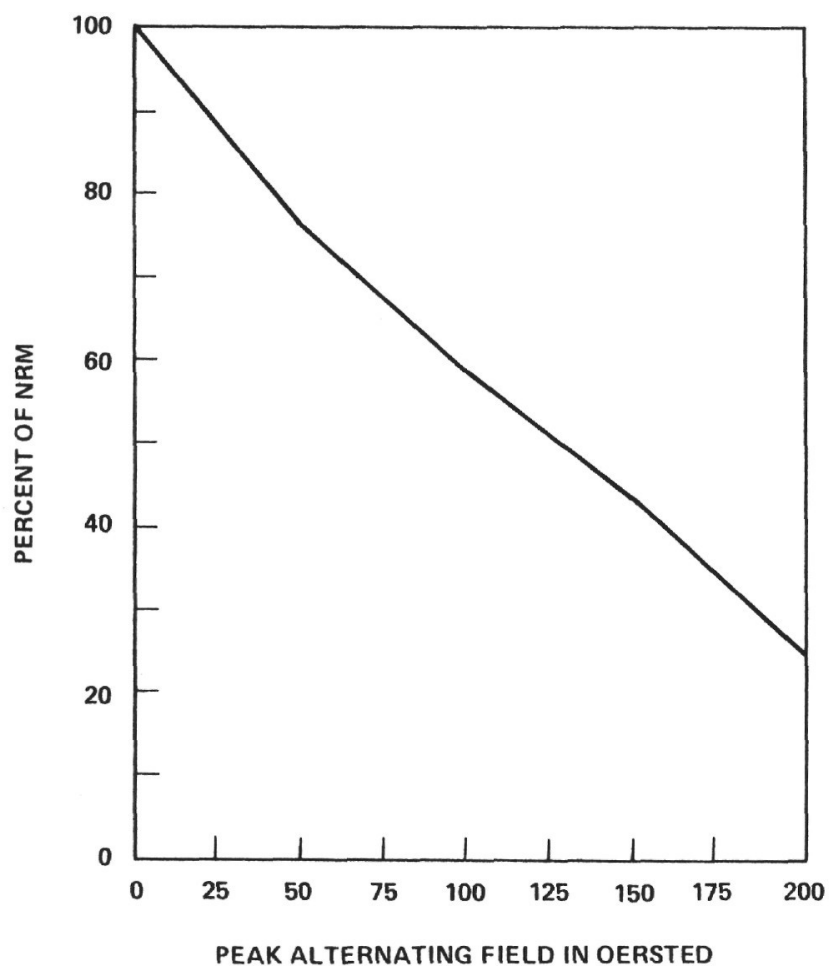

Figure 5. Sample $28-2-2,10 \mathrm{~cm}$.

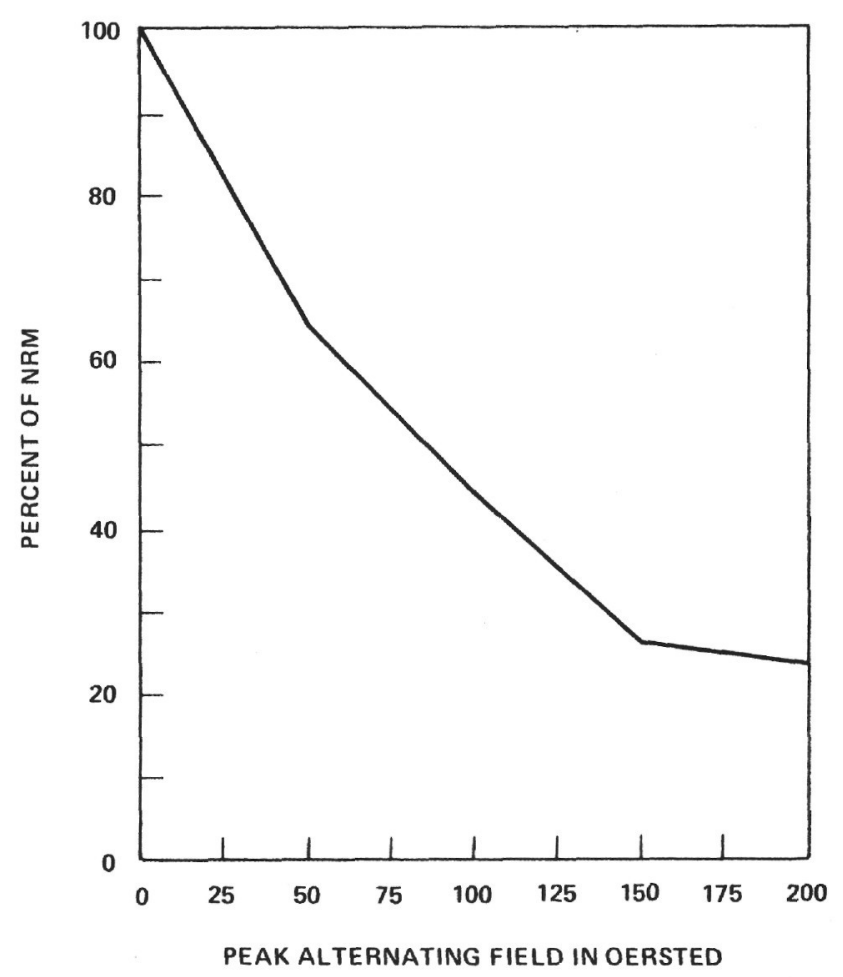

Figure 6. Sample 29-1-3, $30 \mathrm{~cm}$. 


\section{MAGNETIC SUMMARY CHARTS}

Figures 7 through 10

Summary charts of magnetic direction after cleaning in 50 oersteds in terms of depth in hole for each site at which cleaned data was available. Sediment ages are also given. 


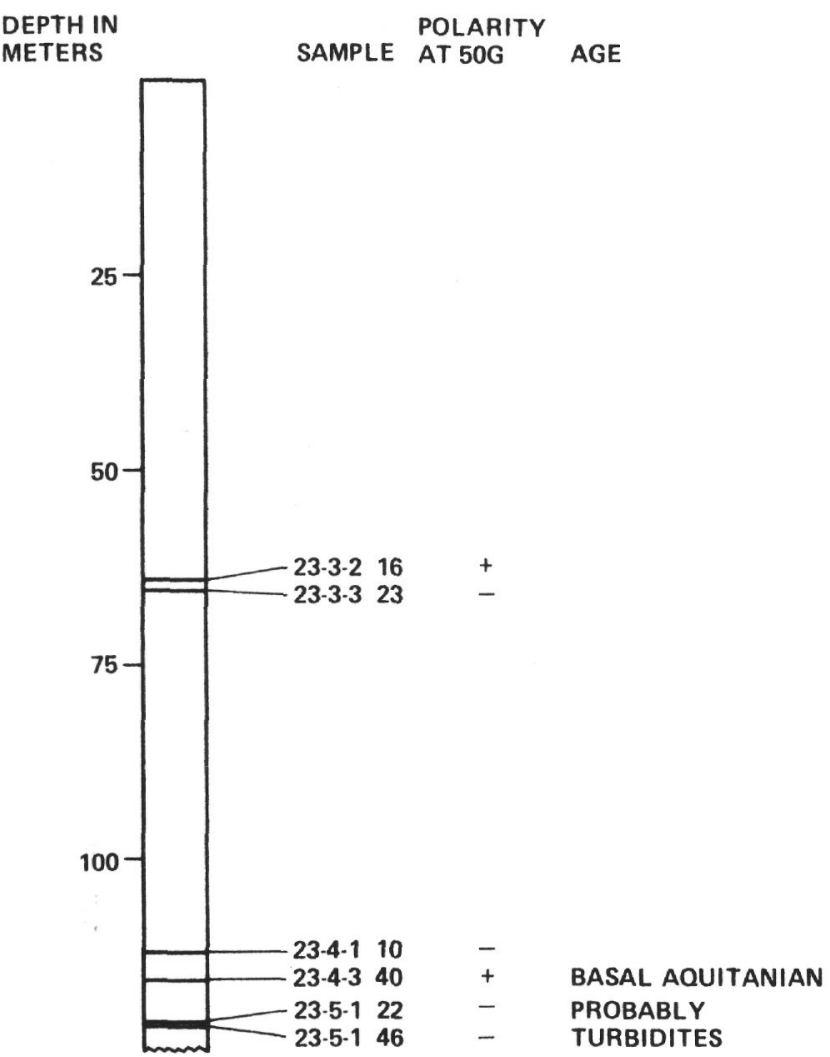

Figure 7. Site 23.

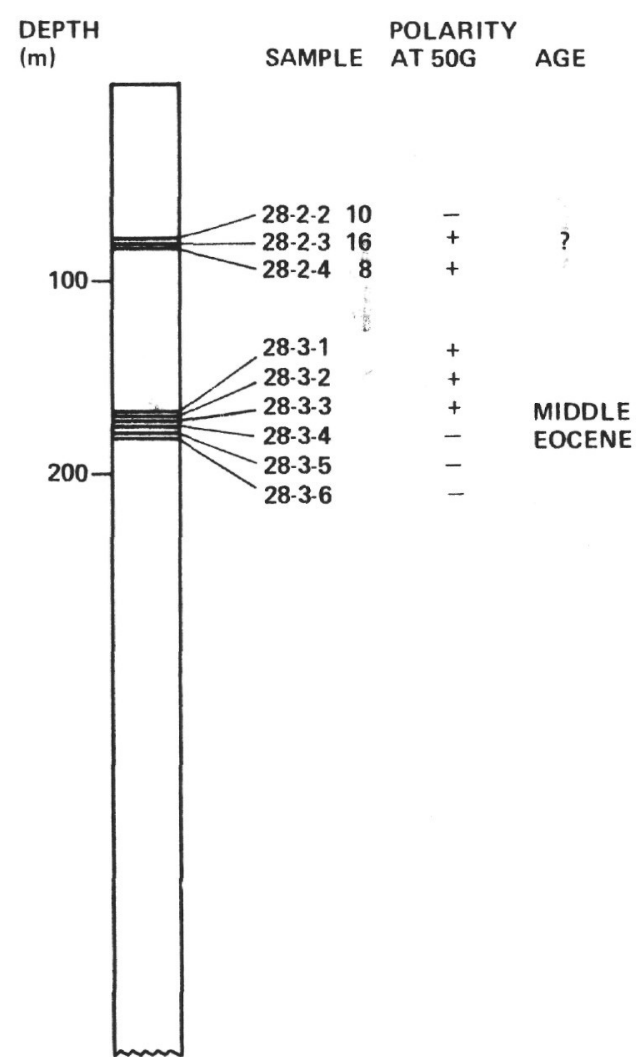

Figure 9. Site 28.

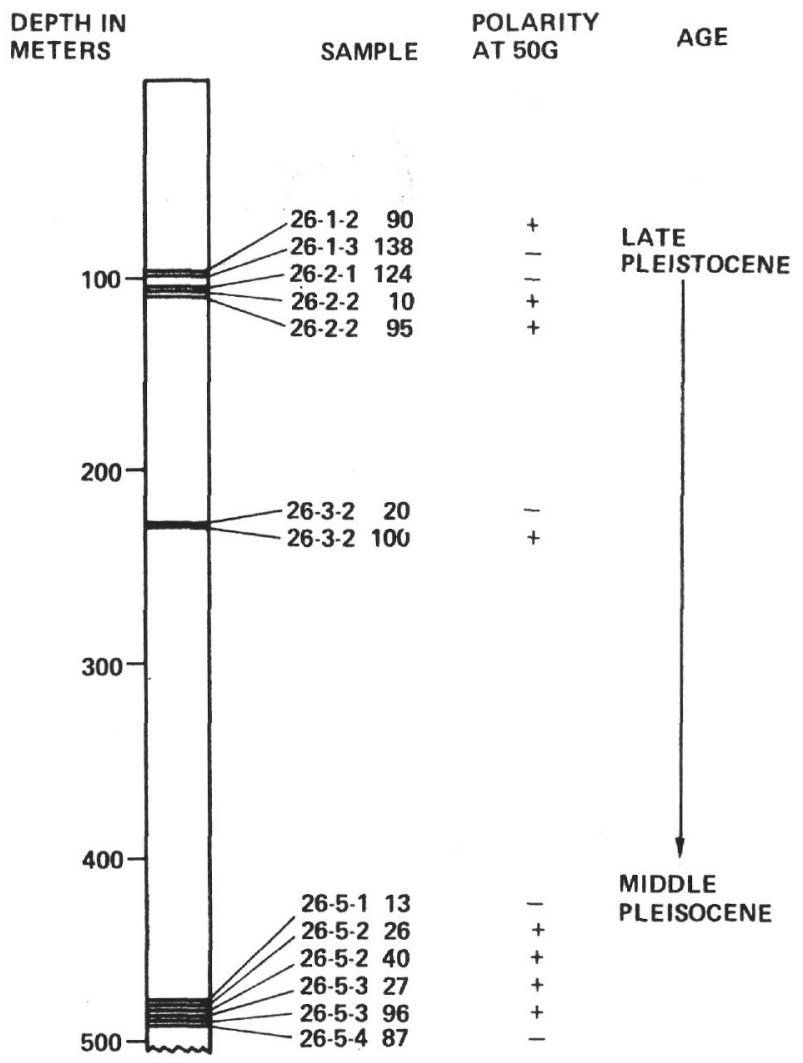

Figure 8. Site 26.

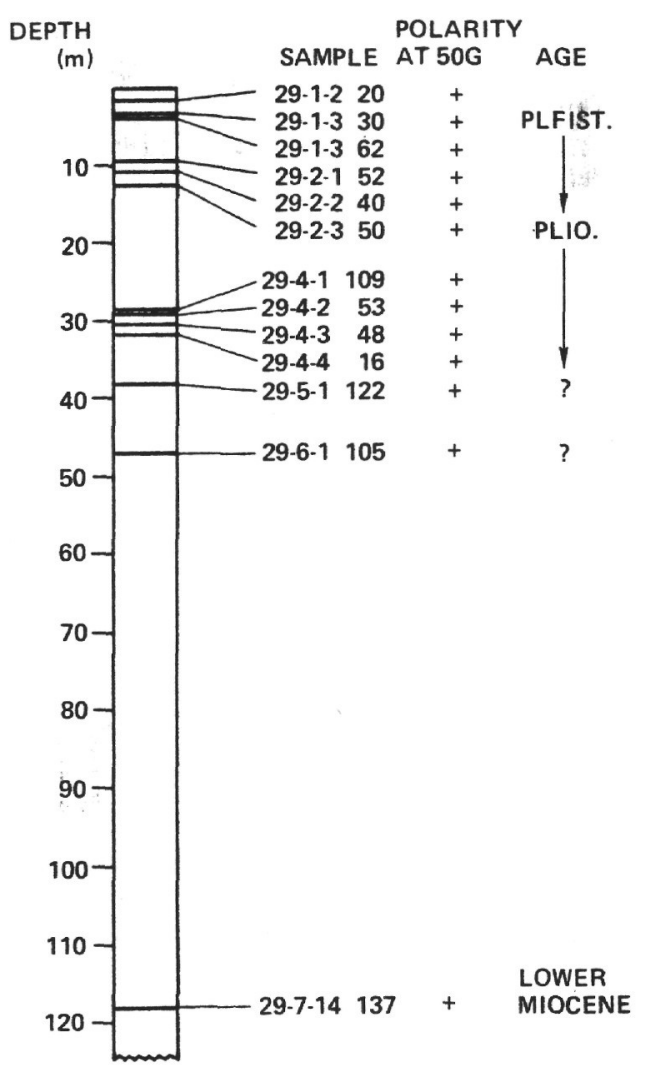

Figure 10. Sites 29 and $29 B$. 


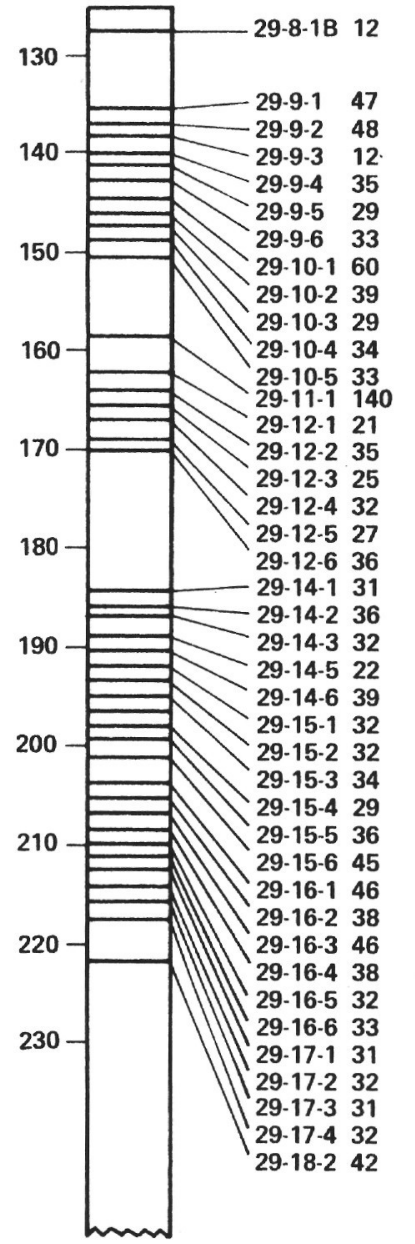

Figure 10. Continued.

UPPER

EOCENE

MIDDLE

EOCENE

$+$

$+$

-

$+$

$+$

$-$

$\pm$

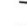

$+$

$+$

$+$

$-$

$+$ 